\title{
High-dose-rate Brachytherapy of Prostate Cancer
}

\author{
a report by \\ D Jeffrey Demanes and Dennis R Hill
}

California Endocurietherapy Cancer Center, Oakland

Measurement of blood levels of prostate-specific antigen (PSA) and greater awareness have led to the detection of prostate cancer at an earlier and potentially more curable stage. As certain kinds of prostate cancer progress very slowly, some patients choose to undergo clinical observation ('watchful waiting') rather than active treatment. Alternatively, surgery and radiation therapy (RT) are the only curative treatment options. Hormone therapy (androgen deprivation) is another intervention used to enhance curative treatment or treat recurrent disease. RT can be administered in various ways, including external beam (EB), brachytherapy (the placement of a radiation source directly into a tumour) or a combination of both methods. Brachytherapy effectively targets the prostate and minimises the dose to adjacent organs such as the bladder and rectum, because the dose decreases very rapidly as the distance from the radiation source increases.

There are two types of prostate brachytherapy: high-dose-rate (HDR) and permanent-seed. HDR brachytherapy is a precision technology for the placement of a brachytherapy source that employs robotic technology to temporarily deliver a high-intensity radiation source into the prostate and surrounding tissue. HDR is applicable to virtually all stages of localised prostate and many other types of cancer. It may be given as the only treatment (HDR monotherapy) for

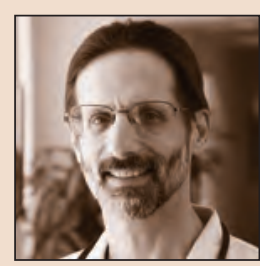

D Jeffrey Demanes founded California Endocurietherapy Cancer Center in 1981. He is on the voluntary clinical staff at Stanford University and provides brachytherapy training for the resident program. He is Chairman of the Board of Chancellors of the American College of Radiation Oncology (ACRO), and was its President from 2005 to 2007. He is also Co-Chair of the Health Policy Committee of the American Society for Therapeutic Radiology and Oncology (ASTRO) and a Fellow of the American College of Radiology (ACR). Dr Demanes is considered a pioneer and leader in the field of highdose-rate brachytherapy, and he is active in clinical outcome studies and actively contributes to the medical literature. He graduated from the University of California, Los Angeles Medical School, where he also completed his residencies in internal medicine and oncology. Dr Demanes is board-certified in three medical specialities: radiation oncology, medical oncology and internal medicine.

E: jeff@cetmc.com

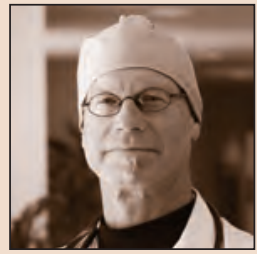

Dennis R Hill joined the California Endocurietherapy (CET) Cancer Center in 2004. His previous positions include Director of Radiation Oncology at the Naval Regional Medical Center in San Diego, Assistant Clinical Professor at the University of California, San Diego, Clinical Professor at the University of California, San Francisco (UCSF) and Vice Chairman of the Department of Radiation Oncology at UCSF. He also practised external-beam radiation in private practice at Davies Medical Center in San Francisco prior to joining CET Cancer Center. Dr Hill graduated from the Oregon Health and Science University and completed his radiation oncology residency at UCSF. He is board-certified in radiation oncology. early disease or used in combination with external RT for locally advanced or higher-stage cancer. This article will describe HDR brachytherapy for prostate cancer.

\section{High-dose-rate Brachytherapy}

HDR describes more than the rate at which the radiation dose is given; it also offers a new dimension to brachytherapy. A single high-intensity radiation source located at the end of a wire is robotically inserted into thin brachytherapy catheters (close-ended straws) that have been temporarily inserted into the prostate. Instead of having a large number of uniform-strength permanent seeds, HDR applies a single radiation source that is time- and position-controlled during the treatment process. The longer the source stays in a particular location, the greater the effective radiation dose. Thus, HDR offers an infinite variety of source strengths at each of the hundreds of locations within the implant.

Along with the millimetre precision of source insertion, HDR provides the best opportunity to shape and control the distribution of the radiation. Delivery of treatment takes only about 20 minutes and occurs in a series of applications given over several hours or days. The radiobiology of prostate cancer favours large doses of radiation per session, thus HDR is well-suited for the control of prostate cancer. Due to the short duration of therapy, HDR has the benefit of a correspondingly short period of acute side effects.

The temporary HDR brachytherapy catheters act as stable scaffolding to permit precise and reproducible placement of the radiation source within and around the prostate gland. Consequently, there is a high degree of dose uniformity within the gland and excellent coverage of disease just beyond the edge of the prostate. Importantly, unlike with permanent-seed brachytherapy, the dose of radiation is known before treatment and can be adjusted and individually tailored. There is no radiation exposure to medical personnel or families and seed migration to other organs cannot occur. Organ motion, of great concern in EBRT, is not a problem with HDR as the applicator is fixed to the target. Rectal doses are predictably low with HDR brachytherapy compared with EBRT.

The four steps of HDR brachytherapy are: ${ }^{1}$ image-guided applicator insertion, ${ }^{2}$ image acquisition of the completed implant (simulation radiography), ${ }^{3}$ dose distribution calculation (computerised dosimetry) ${ }^{4}$ and treatment delivery. ${ }^{1,2}$ The initial placement of thin, hollow, closedended catheters is performed under image guidance (ultrasound, computed tomography [CT] or magnetic resonance imaging [MRI]) and cystoscopy. The radiation oncologist and the urologist usually perform the outpatient applicator insertion procedure together, combining their knowledge and skills to achieve optimal placement. 
The radiation oncology team consists of a brachytherapy nurse, a dosimetrist, a medical physicist and radiation therapists. The images of the final implant position are acquired and downloaded into a treatment planning computer to create a virtual image of the implant and surrounding normal structures. The treatment planning computer is then used to calculate a unique patient-specific 3D dose distribution. The doses are viewed as isodose curves overlaid on an image such as a CT scan or ultrasound, compared as volume graphs (dose volume histograms) and represented as a 3D virtual image of the prostate tumour target and surrounding structures (see Figure 1). Once dosimetry calculations are completed, the instructions on where the source should be positioned within the implant catheters and for how long are sent to the robotic delivery device called the 'remote afterloader' (see Figure 2). Treatment is delivered under realtime source position monitoring. As the source is removed after treatment there is no residual radiation or radioactivity and the applicator is removed before the patient goes home.

HDR may be given in one or a series of implants (catheter placement procedures) with one or more treatments (radiation source delivery sessions called 'fractions') per implant. HDR prostate treatments are often given twice daily. The total number and sequence may vary between treatment centres depending in part on whether or not EBRT is also administered. Because there is no incision and no surgical wound to heal, recovery is rapid. Temporary urinary frequency and urgency are expected for one to two weeks after the implant. Urinary outflow problems occur more often in patients with symptomatic benign prostate hyperplasia (BPH). They are generally self-limited and best managed conservatively.

\section{California Endocurietherapy Cancer Center Protocols}

The treatment protocols described in this presentation were derived from ongoing long-term studies of patients in various risk groups treated at the California Endocurietherapy (CET) Cancer Center. The CET risk group classification is shown in Table 1. The protocols consist of two implant procedures performed approximately one week apart. Low-risk and 'favourable' intermediate-risk group patients are treated with HDR monotherapy (two brachytherapy procedures and a total of six HDR treatments without EBRT). Patients who are in the 'less favourable' intermediate- or high-risk groups are treated with a combination of HDR (two brachytherapy procedures and a total of four HDR treatments) and moderate-dose EBRT.

The intention of HDR monotherapy is to confine the curative dose of radiation to the prostate and immediate surrounding tissue and to keep normal tissue injury to a minimum. For more extensive disease, a larger area should be treated with EBRT, and HDR brachytherapy is used to safely deliver the high dose to the primary tumour. Dividing the implants into two separate sessions with multiple treatments allows for recovery of normal tissue and lessens the likelihood of normal tissue injury.

\section{California Endocurietherapy High-dose-rate}

\section{Brachytherapy Results}

Approximately 1,700 patients with prostate cancer have been treated at the CET Cancer Center since 1991. Our outcome studies have confirmed that HDR brachytherapy is a safe and effective treatment for prostate cancer. Long-term outcome results from the first 209 patients treated with HDR and EBRT (no androgen deprivation) were published

\section{Figure 1: 3D Virtual Image of the Pelvic Anatomy}

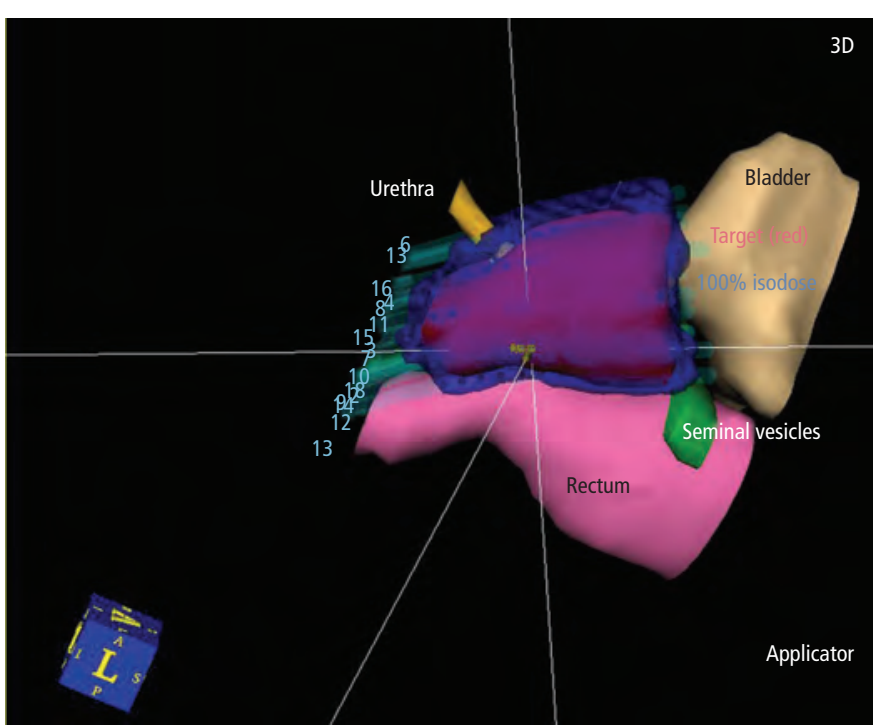

The prostate (dark red) is covered by the radiation isodose cloud (blue).

Figure 2: Afterloaders
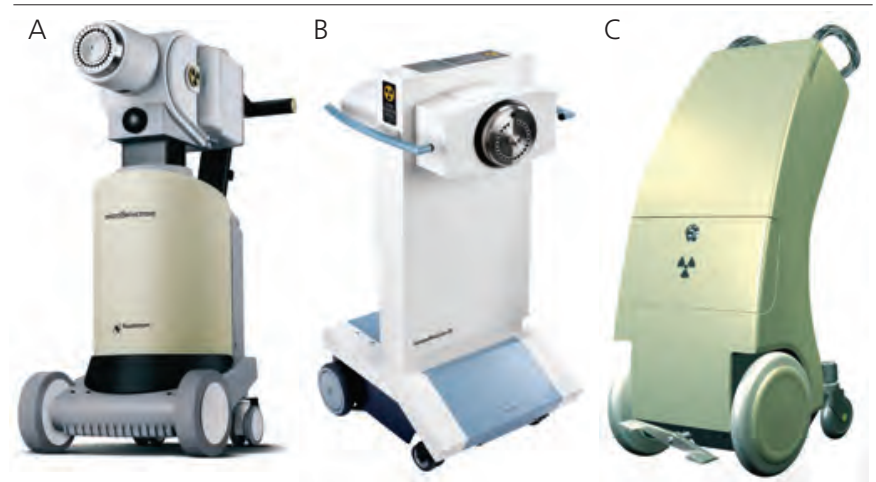

A: The microSelectron V2 afterloader Nucletron Corporation (www.nucletron.com). B: The GammaMed afterloader Varian Medical Systems (www.varian.com). C: The Flexitron afterloader Isodose Control BV (www. isodosecontrol.com).

Table 1: California Endocurietherapy Risk Group Classification

\begin{tabular}{lllll} 
Risk Group & T-stage & PSA & Gleason & Grade Rules \\
\hline Low & T1-T2a & $\leq 10$ & $\leq 6$ & All required \\
\hline Intermediate & T2bc & $>10-\leq 20$ & 7 & One or more \\
\hline High & T3 & $>20$ & $8-10$ & One or more \\
\hline
\end{tabular}

in 2005. ${ }^{3}$ General clinical control (no clinical or PSA evidence of disease) was achieved in $90 \%$ of cases and the cause-specific survival was $97 \%$. The PSA control rates stratified by risk group were $90 \%$ for low-risk patients, $87 \%$ for intermediate-risk patients and $69 \%$ for high-risk patients. There were no statistically significant differences in outcome between the low- and intermediate-risk group patients. Complication rates were low $(2 \%$ grade $1,2 \%$ grade 2 rectal complications and $0 \%$ grade 3 or 4 rectal complications). The $7.7 \%$ grade 3-4 urinary effects were consistent with other forms of RT. These results are among the best reported in the literature. We also reported results in $2005^{4}$ on a total of 411 patients from the same period comparing patients who did or did not receive androgen deprivation therapy (hormone treatment). There was no difference in outcome identified in patients who received androgen deprivation therapy. The PSA and clinical control rates after 10 years of follow-up 


\section{Prostate Cancer}

Table 2: High-dose-rate plus External-beam Radiation Therapy in Low-risk Patients

\begin{tabular}{|c|c|c|c|c|c|}
\hline Author (year) & $\mathrm{n}$ & HDR & EBRT & $\mathrm{F} / \mathrm{U}$ & PSA-PFS (\%) \\
\hline Eulau-Mate, $2000^{7}$ & 40 & $3-4$ Gy x 4 & 50Gy & 6 & 96 \\
\hline Galalae, $2002^{8}$ & 46 & $9 \mathrm{~Gy} \times 2$ & 40Pr/50Pelv & 5 & 96 \\
\hline Pellizzon, $2003^{9}$ & 55 & $4-5$ Gy $x 4$ & $45 \mathrm{~Gy}$ & 3.5 & 73 \\
\hline Neumann, $2005^{10}$ & $\mathrm{n} / \mathrm{a}$ & $5.5-6.75 \mathrm{~Gy} \times 4$ & $45 G y$ & 5 & 89 \\
\hline Demanes, $2005^{4}$ & 110 & $5.5-6 \mathrm{~Gy} \times 4$ & $36-40 \mathrm{~Gy}$ & 7 & 93 \\
\hline Phan, $2005^{11}$ & 67 & $6 \mathrm{~Gy} \times 4$ & 40-50Gy & 5 & 98 \\
\hline Yamada, 200612 & 15 & $5.5-7 \mathrm{~Gy} \times 3$ & 45-50Gy & 3.5 & 100 \\
\hline
\end{tabular}

Table 3: High-dose-rate plus External-beam Radiation Therapy in Intermediate- and High-risk Patients ( $<5$-year Follow-up)

\begin{tabular}{llllllr} 
Author (year) & Group & $\mathrm{n}$ & HDR & EBRT & F/U & $\begin{array}{r}\text { PSA-PFS } \\
(\%)\end{array}$ \\
\hline Borghede, 199714 & Interm. + high & 50 & $10 \mathrm{~Gy} \times 2$ & 50 & 4 & 84 \\
\hline Martinez, 2002 & Low-dose & 58 & $5.5-6.5 \mathrm{~Gy} \times 3$ & 46 & 4 & 52 \\
& High-dose & 149 & $8-11.5 \mathrm{~Gy} \times 2$ & & & 87 \\
\hline Pellizzon, 20039 & Interm. + high & 53 & $4-5 \mathrm{~Gy} \times 4$ & 45 & 3.5 & 66 \\
\hline Hsu, 200515 & Interm. + high & 64 & $6 \mathrm{~Gy} \times 3$ & 45 & 4 & 92 \\
\hline Yamada, 2006 32 & Intermediate & 62 & $5.5-7 \mathrm{~Gy} \times 3$ & $45-50$ & 3.5 & 98 \\
& High & 28 & & & & 92 \\
\hline
\end{tabular}

were $93 \%$ for the low-risk group, $87 \%$ for the intermediate-risk group and $70 \%$ for the high-risk group. Local tumour control was $98 \%$ across all risk groups. These findings bring into question the need for androgen deprivation therapy when high doses of radiation are given to the prostate and surrounding tissue.

Based on the favourable experiences with both HDR and permanent-seed brachytherapy without EBRT in early-stage disease, we started HDR monotherapy for low- and intermediate-risk group patients ( $\mathrm{T} 1$ or $\mathrm{T} 2$, PSA $<15$ and Gleason $\leq 7$ ). We accomplished control of disease in $96 \%$ of cases (PSA progression-free survival) and observed a very low complication rate. ${ }^{5}$ To confirm that the EBRT was not needed, we performed a matched-pair analysis that compared the results of patients who received HDR and EBRT with the results of those who received HDR monotherapy. ${ }^{6}$ HDR monotherapy was as effective as the combined treatment programme, so we concluded that for early cancer of the prostate HDR monotherapy is effective and ample treatment.

\section{Published Literature}

Table 2 shows the results from the literature of HDR in combination with EBRT in patients with low-risk disease. The mean PSA progression-free survival (survival with no clinical, radiological or PSA signs of disease progression) was over $90 \%$, with most patients having five or more years of follow-up.

Tables 3 and 4 show HDR brachytherapy and EBRT outcomes in patients with intermediate- and high-risk disease based on the length of followup. The definition of intermediate- and high-risk patients varies in the prostate literature. This is significant as upgrading patients to high-risk can exaggerate the efficacy of therapy. Regardless of this, landmark studies by Martinez et al. ${ }^{13}$ revealed that dosage dramatically affects tumour control. Patients who received the higher doses had an $87 \%$ control rate (PSA progression-free survival) compared with $52 \%$ in patients who received lower doses.
Table 4: High-dose-rate plus External-beam Radiation Therapy in Intermediate- and High-risk (<5-year Follow-up)

\begin{tabular}{|c|c|c|c|c|c|c|}
\hline Author (year) & Group & $\mathrm{n}$ & HDR & EBRT & $\mathrm{F} / \mathrm{U}$ & $\begin{array}{r}\text { PSA-PFS } \\
(\%)\end{array}$ \\
\hline \multirow[t]{2}{*}{ Eulau-Mate, $2000^{7}$} & Intermediate & 42 & $3-4 G y \times 4$ & 50 & 6 & 72 \\
\hline & High & 22 & & & & 49 \\
\hline \multirow[t]{2}{*}{ Galalae, $2002^{8}$} & High +19 & 144 & 15Gy x 2 & 40 & 8 & 73 \\
\hline & Intermediate & & & & & \\
\hline \multirow[t]{2}{*}{ Demanes, $2005^{4}$} & Intermediate & 188 & $5.5-6$ Gy $\times 4$ & 36 & 7 & 86 \\
\hline & High & 113 & & & & 71 \\
\hline Neumann, $2005^{10}$ & Definition n/a & n/a & $5.5-6.75 G y \times 4$ & 45 & 5 & 89 \\
\hline \multirow[t]{2}{*}{ Phan, $2005^{11}$} & Intermediate & 109 & $6 \mathrm{~Gy} \times 4$ & $40-{ }^{-} 50$ & 5 & 9 \\
\hline & High & 133 & & & & 78 \\
\hline
\end{tabular}

Table 5: Results from Multi-institutional Studies

\begin{tabular}{llllllr} 
Author (year) & Group & $n$ & HDR & EBRT & F/U & $\begin{array}{r}\text { PSA-PFS } \\
(\%)\end{array}$ \\
\hline Martinez, 2005 & & & & & & 85 \\
& Gleason $\geq 7$ or & 934 & Variable & $36-50$ & 4 & \\
& PSA $\geq 10$ or & & & & & \\
& Stage $\geq$ T2b & & & & & \\
\hline Vargas, 200517 & Roach $<15 \%$ & 761 & Variable & $36-50$ & 4 & 83 \\
& Roach $>15-30 \%$ & 422 & & & & 68 \\
& Roach $>30 \%$ & 174 & & & & 67 \\
\hline Swanson, 2006 & Gleason 8-10 & 313 & Variable & $36-50$ & 5 & \\
\hline
\end{tabular}

Roach formula: (Gleason grade - 6) × $10+2 / 3 P S A=$ per cent risk of $L N$ involvement.

Table 5 shows the results of multi-institutional HDR studies performed to evaluate the benefits of treatment of androgen deprivation (hormone treatment) and radiation of pelvic lymph nodes. Neither a short course ( $<6$ months) of androgen deprivation nor irradiation of pelvic lymph nodes was demonstrated to be of additional benefit. ${ }^{16-18}$ However, they confirmed the excellent results of HDR brachytherapy across all risk groups, including patients with Gleason grades 8-10.18

The results of HDR monotherapy (HDR given without EBRT) for low-risk and intermediate-risk groups is presented in Table 6.24-26 Our study of 298 patients from the CET and William Beaumont Hospital demonstrated a fiveyear control rate of $94 \%$ and a cause-specific survival rate of $100 \%$, and there were no patients with distant metastasis. The rectal complication rates were $<1 \%$ and there were $<5 \%$ grade 3 urinary complications.

Advantages and Comparisons of

High-dose-rate Brachytherapy

High-dose-rate Brachytherapy Is Intensity-modulated HDR is 'intensity-modulated' (IM) because the longer the HDR radiation source resides in a particular location, the greater the intensity of radiation at that location. Modulating the intensity comes from adjusting the time the source stays at each position within the catheter matrix. It is the functional equivalent of having an infinite range of seed activity at every possible source position, so offers a new level of refinement in dose distribution control. IM HDR brachytherapy does not have the patient set-up and prostate motion problems associated with external-beam IMRT. HDR is delivered with great precision. The single high-intensity HDR source is robotically delivered into stable catheters with millimetre precision. The HDR source does not shift or migrate. Permanent seeds, in contrast, are manually inserted into deformable soft tissue and the final locations of the sources are usually, to some degree, different from those planned. Even if an ideal seed 
implant were accomplished, prostate swelling and seed migration after insertion may result in suboptimal dosimetry.

High-dose-rate Dosimetry Is Dynamic and Prospective The distribution of the HDR implant catheters and dwell positions are known from 3D imaging and the dosimetry can be adjusted in advance of the source delivery. With permanent seeds, needle and source insertion are contemporaneous and the radiation sources are supposed to be static. The seed dose is determined retrospectively and cannot be modified. HDR brachytherapy differs from seed brachytherapy because the final HDR dosimetry is completed and approved by the physician before rather than during or after the administration of the source.

The Relationship of the Implant to the Patient's Anatomy Is Known and Maintained During the High-dose-rate Source Delivery

An ideal radiation delivery system should establish and maintain the anatomical relationships to the target and the adjacent normal tissues throughout treatment. Permanent-seed and EBRT doses can differ

High-dose-rate (HDR) is 'intensitymodulated' because the longer the HDR radiation source resides in a particular location, the greater the intensity of radiation at that location.

substantially from the planned dose because of anatomical changes during the time it takes a permanent-seed implant to emit the dose or through patient motion or organ deformation during the course of EBRT. In contrast, the precise sequence of HDR simulation, dosimetry and dose delivery, in conjunction with the short treatment duration, permits accurate pre-treatment dosimetry representations and reliable treatment delivery. Correct and reproducible geometric plan-to-target and sourceto-target relationships are achieved with HDR brachytherapy.

The High-dose-rate Sequence of Events Allows Multiple Opportunities to Optimise Therapy

Inevitably, in some cases brachytherapy needle or catheter placement will be suboptimal. The sequencing of HDR permits the physician to discover and improve suboptimal positioning and dosimetry either by moving the catheters to better positions or by utilising computer software to optimise the dosimetry. In addition, relatively large prostates can be treated with HDR due to the flexibility of the catheter matrix system.

\section{High-dose-rate Reliably Treats Cancer That}

\section{Extends Beyond the Prostate}

HDR brachytherapy's 'scaffolding matrix' feature provides both general stability and the ability to place catheters at or beyond the prostate capsule and into the seminal vesicles without the possibility of source loss or migration to other organs. Figure 3 shows the $100 \%$ therapeutic isodose extending beyond the prostate capsule. Our high local tumour control rates in all risk groups confirm the efficacy of HDR brachytherapy to control disease both in and around the prostate. ${ }^{3-5}$
Table 6: Results of High-dose-rate Monotherapy

\begin{tabular}{lllllr} 
Author (year) & $\mathrm{n}$ & HDR & EBRT & F/U & PSA-PFS (\%) \\
\hline Yoshi, 2003 & 15 & 6 Gy $\times 9$ & None & 2 & $86^{*}$ \\
\hline Mark, 200720 & 193 & $7.5 G y \times 6$ & None & 6 & 89 \\
\hline Demanes, 200721 & 298 & $\begin{array}{l}7.25 G y \times 6 \text { or } \\
\text { 9.5Gy x 4 }\end{array}$ & None & 3.9 & 94 \\
\hline
\end{tabular}

* Low- and intermediate-risk patients only.

Figure 3: Computed Tomography Cross-section of the Pelvis

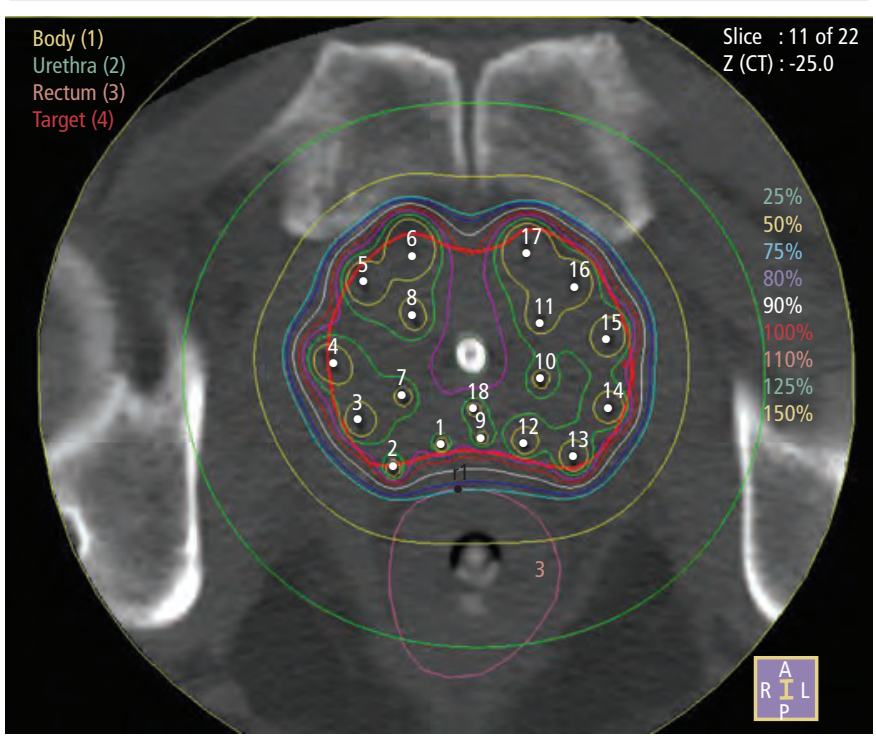

With implant catheter positions in the prostate and the radiation isodose curves covering the target.

Control of Normal Tissue Doses Is Predictable with High-dose-rate Brachytherapy

The dose to the bladder and rectum is routinely kept within prescribed constraints (typically $75-80 \%$ at CET). ${ }^{4}$ It is a subtle but significant fact that at larger fraction sizes the biological differences are more pronounced than the simple percentages would imply. Thus, the biological doses to the bladder and rectum are actually lower than the nominal percentages. Dose limits are readily achieved by avoiding the urethra and rectum during catheter insertion and by making dwell time adjustments during the dosimetry calculations.

Radiobiological Advantages for High-dose Radiation in the Treatment of Prostate Cancer

It has been established that the repair of sub-lethal radiation damage for prostate cancer is comparatively lower than previously believed.22-27 It suggests that the large doses per session and the relatively short time course confer an important biological advantage to HDR brachytherapy.

\section{Radiation Safety Is Excellent}

Radiation exposure to people other than the patient does not occur with HDR brachytherapy. Complicated permanent-seed accounting measures are not relevant to HDR. There is no seed loss or environmental exposure.

High-dose-rate Causes a Comparatively Short

Period of Acute Symptoms

All forms of RT cause transient acute inflammation of pelvic structures and temporary irritation of bowel or bladder function. The duration of these symptoms depends on the time taken to deliver the radiation, total dosage 
of radiation and the volume of tissue irradiated. HDR brachytherapy limits the time, dose and volume of radiation to normal structures so that the

High-dose-rate is appealing because

the source both is reusable and can

be applied to many patients and

many different kinds of cancer other

than prostate.

symptoms are of relatively short duration - two to three weeks compared with those resulting from approximately two months of treatment during EBRT or after permanent-seed brachytherapy.

\section{High-dose-rate Has Low Chronic Rectal and}

\section{Urinary Complication Rates}

Side effects of prostate irradiation are related to the total dose and the volume of the normal structures irradiated. By using the gradient effect of brachytherapy to limit the dose to surrounding structures, complication rates can be kept to a minimum. In the case of low- or intermediate-risk group cases, the use of HDR monotherapy (brachytherapy without EBRT) can minimise normal tissue injury. For example, rectal complication rates with combined HDR and EBRT at CET were $2 \%$ grade 1 and $2 \%$ grade $2,{ }^{4}$ and for HDR monotherapy $<1 \% .{ }^{21}$ Similarly, chronic urinary side effects occur in $<10 \%$ of cases and depend on underlying symptomatic benign disease and prior urinary surgeries. ${ }^{4}$ Conservative measures such as medication and avoidance of urinary surgery after RT are important for limiting longterm complications. The rates of urinary incontinence for patients who do not have surgical intervention are $<1 \%$.

\section{High-dose-rate Is a Reusable Resource}

HDR is appealing because the source both is reusable and can be applied to many patients and many different kinds of cancer other than prostate. The HDR source is potentially available, even in remote areas, without the logistics of source inventory or acquisition.

\section{Follow-up Medical Care and Prostate-specific Antigen Testing After Therapy}

Most patients are back to baseline status within one month of treatment. Subsequent PSA testing (every three months for two years and every six months thereafter) at the CET requires correct interpretation because transient risings (called PSA 'spikes' or 'bounces') may occur and should not be misinterpreted as treatment failures. ${ }^{28}$ Biopsies within two years of treatment are frequently unreliable indicators of persistent disease.

\section{Conclusions}

HDR brachytherapy is a safe and effective treatment of localised prostate cancer. It combines the best characteristics of permanent-seed brachytherapy and the intensity modulation that typifies external-beam IMRT. It has great precision and is a dynamic process that permits excellent control of radiation dosimetry and treatment delivery. The relationship of the implant and dose distribution to patient anatomy are known in advance of treatment so the dosimetry is prospective and hence modifiable. HDR reliably treats local extension of disease beyond the prostate. Normal tissue dose constraints are readily attainable, predictable and reliable. There is a radiobiological advantage to the HDR fractionation and the accelerated treatment course. Radiation safety is optimal. Acute radiation side effects are of short duration and chronic effects are relatively few. HDR may be safely and effectively applied to all risk groups either as monotherapy for early to intermediate disease or in combination with EBRT for intermediate to high disease. It can also be safely and effectively applied to patients with larger glands, often without the need for hormone therapy. As with all sophisticated technologies, the accuracy of the technique depends on when and how it is used.
1. Demanes $D$, Rodriguez $R$, Altieri $G$, High-dose brachytherapy: the California Endocurietherapy (CET) Method, Radiother Oncol, 2000;57:289-96.

2. Stromberg J, Martinez A, Gonzalez J, et al., Ultrasound-guided high dose rate conformal brachytherapy boost in prostate cancer: Treatment description and preliminary results of a phase I/II clinical trial, Int I Radiat Oncol Biol Phys, 1995;33:161-71.

3. Demanes $D$, Rodriguez $R$, Schour $L$, et al., High dose rate intensity-modulated brachytherapy with external beam radiotherapy for prostate cancer: California Endocurietherapy's 10-year results, Int I Radiat Oncol Biol Phys, 2005;61:1306-16.

4. Demanes $D$, Altieri $G$, Brandt $D$, et al. Long-term results of high dose rate brachytherapy and external beam with and without androgen suppression for prostate cancer, Int I Radiat Oncol Biol Phys, 2005;63:S38.

5. Schour L, Demanes, J, Altieri G, et al., High Dose Rate Monotherapy for Prostate Cancer, Int J Radiat Oncol Biol Phys, 2005;(Suppl. 1):S315.

6. Matched Pair analysis 117, ASTRO Annual Meeting, 2006.

7. Eulau SM, Hollebeke L, Cavanagh W, et al., High dose rate Iridium 192 brachytherapy in localised prostate cancer: Results and toxicity with maximum follow-up of 10 years, Int I Radiat Oncol Biol Phys, 2000;48:149.

8. Galalae R, Kovacs G, Schultze J, et al., Long-term outcome after elective irradiation of the pelvic lymphatics and local dose escalation using high dose rate brachytherapy for locally advanced prostate cancer, Int I Radiat Oncol Biol Phys, 2002; 52:81-90

9. Pellizzon A, Nadalin W, Salvajoli J, Results of high dose rate afterloading brachytherapy boost to conventional external beam radiation therapy for initial and locally advanced prostate cancer, Radiother Oncol, 2003;66(2):167-72.
10. Neumann T, Mark R, Akins S, Nair M, Interstitial High Dose Rate (HDR) Brachytherapy + IMRT versus HDR Monotherapy for Early Stage Prostate Cancer, Int I Radiat Oncol Biol Phys, 2005;63:S313.

11. Phan T, Puthawala A, Sharma A, Syed A, Treatment of localised prostate cancer with external beam radiation therapy and high dose rate interstitial brachytherapy, Int I Radiat Oncol Biol Phys, 2005;63:\$315.

12. Yamada Y, Bhatia S, Zaider M, et al., Favourable clinical outcomes of three-dimensional computer-optimised high dose rate prostate brachytherapy in the management of localised prostate cancer, Brachytherapy, 2006;5(3):157-64.

13. Martinez AA, Gustafson G, Gonzalez J, et al. Dose escalation using conformal high dose rate brachytherapy improves outcome in unfavourable prostate cancer, Int I Radiat Oncol Biol Phys, 2002;53:316-27.

14. Borghede, G, Hedelin $H$, Holmang $S$, et al., Combined treatment with temporary short-term high dose rate Iridium-192 brachytherapy and external beam radiotherapy for irradiation of localised prostatic carcinoma, Radiother Oncol, 1997;44:237-44.

15. Hsu I, Cabrera A, Weinberg V, et al., Combined modality treatment with high dose rate brachytherapy boost for locally advanced prostate cancer, Brachytherapy, 2005;4:202-6.

16. Martinez A, Demanes DJ, Galalae R, et al., Lack of benefit from short course of androgen deprivation for unfavourable prostate cancer patients treated with an accelerated hypofractionated regime, Int I Radiat Oncol Biol Phys, 2005;62:1322-31.

17. Vargas C, Galalae R, Demanes DJ, et al., Lack of benefit of pelvic radiation in prostate cancer patients with a high risk of positive pelvic lymph nodes treated with high-dose radiation, Int J Radiat Oncol Biol Phys, 2005;63:1474-82.

18. Swanson T, et al., ASTRO Annual Meeting, 2007.

19. Yoshioka Y, Nose T, Yoshida K, et al., High dose rate brachytherapy as monotherapy for localised prostate cancer: a retrospective analysis with special focus on tolerance and chronic toxicity, Int J Radiat Oncol Biol Phys, 2003;56:213-20.

20. Mark R, Paul J, Anderson P, et al., Interstitial high dose rate (HDR) brachytherapy for early stage prostate cancer; A report of 193 cases, Brachytherapy, 20007;6:85-6.

21. Demanes J, et al., ASTRO Annual Meeting, 2007.

22. Duchesne GM, Peters $L$, What is the $\alpha / \beta$ ratio for prostate cancer? Rationale for hypofractionated high dose rate brachytherapy, Int J Radiat Oncol Biol Phys, 1999;44:747-8

23. Fowler J, Chappell R, Ritter $M$, Is $\alpha / \beta$ for prostate tumours really low?, Int I Radiat Oncol Biol Phys, 2001;50:1021-31.

24. Orton C, High dose rate brachytherapy may be radiobiologically superior to low dose rate due to slow repair of late-responding normal tissue cells, Int J Radiat Oncol Biol Phys, 2001;49: 183-9.

25. Brenner DJ, Martinez AA, Edmundson GK, et al., Direct evidence that prostate tumours show high sensitivity to fractionation (low $\alpha / \beta$ ) ratio), similar to late-responding normal tissue, Int J Radiat Oncol Biol Phys, 2002;52:6-13.

26. Brenner DJ, Hypofractionation for prostate cancer radiotherapy - What are the issues?, Int I Radiat Oncol Biol Phys, 2003;57: 912-14.

27. Wang J, Li X, Yu C, DiBiase $S$, The low $\alpha / \beta$ ratio for prostate cancer: What does the clinical outcome of HDR brachytherapy tell us?, Int J Radiat Oncol Biol Phys, 2003;57:1101-8.

28. Horwitz E, Levy LA, Martinez A, et al., The Post-treatment PSA Bounce for Prostate Cancer Patients Treated With External Beam RT or Permanent Brachytherapy Alone Is Not Biochemically or Clinically Significant: A Multi-Institutional Pooled Analysis of More Than 7,500 Patients, Int I Radiat Oncol Biol Phys, 2006;66(Suppl. 1):S205. 
We invite you to visit our

The Multinational

Association of Supportive Care in Cancer (MASCC), a multinational, multidisciplinary organization, is dedicated to research and education in all aspects of supportive care for people with cancer worldwide regardless of the stage of their disease.

website, www.mascc.org, to find out about membership, learn about the organization, visit the Education and Research Centers, and to register for our annual international meeting in Houston, Texas, June 26-28, 2008.

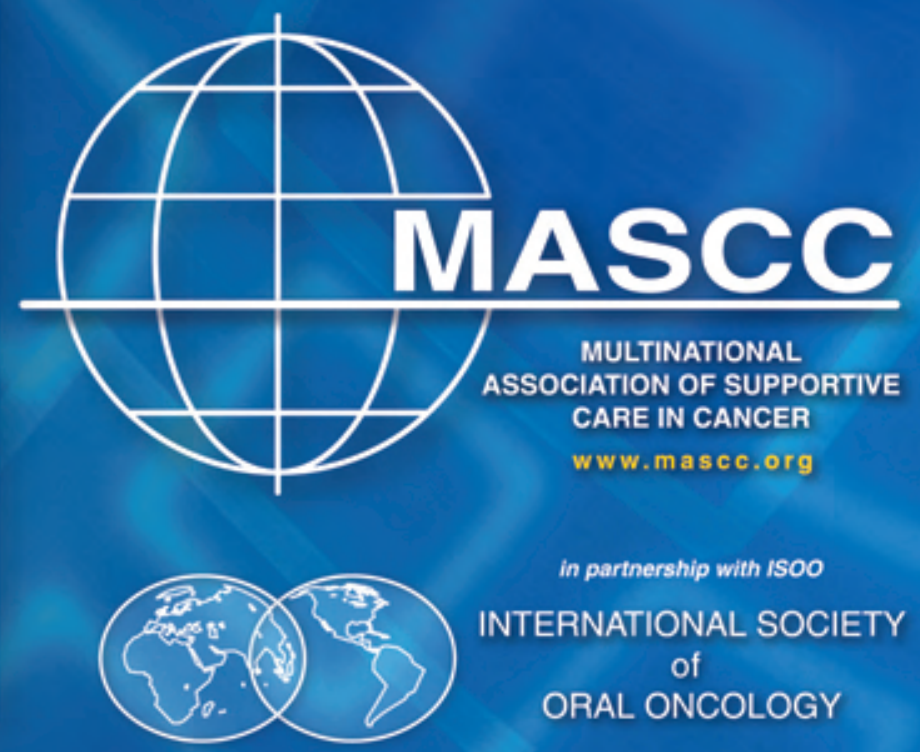

To find out more about MASCC and the Journal of Supportive Care in Cancer please contact Cindy Rittenberg via e-mail at cindyrit@bellsouth.net or visit the website at www.mascc.org 